\title{
Ensino sobre dermatite associada à incontinência subsidiado pela metodologia da problematização
}

\author{
Teaching about incontinence-associated dermatitis informed by problematization methodology \\ La enseñanza sobre dermatitis asociada a la incontinencia subsidiada por la metodología de la \\ problematización
}

\author{
Jaqueline Aparecida dos Santos Sokem'; Fabiana Perez Rodrigues Bergamaschi"; \\ Elaine Aparecida Mye Takamatu Watanabe ${ }^{\prime \prime \prime}$
}

\begin{abstract}
RESUMO
Objetivo: avaliar de modo formativo um processo de intervenção educativa sobre dermatite associada à incontinência com profissionais de enfermagem de um hospital público. Método: estudo de intervenção educativa, com abordagem qualitativa, realizado em 2017, com sete profissionais da equipe de enfermagem do setor de clínica médica, de um hospital universitário da Região Centro-Oeste do Brasil. Investigada amostra não probabilística por conveniência. Os encontros foram gravados na sua totalidade em áudio e para tratamento dos dados utilizou-se a técnica de análise de conteúdo. Estudo aprovado por Comitê de Ética em Pesquisa. Resultados: nos depoimentos foram identificadas as seguintes categorias temáticas: curiosidade epistemológica, pesquisa/investigação, avaliação e alegria. Conclusão: os participantes relataram ter compreendido o tema, bem como afirmaram que a condução dos encontros ocorreu de forma eficiente, alegre e dinâmica. Destaca-se que os profissionais realizaram discussão sobre o problema, mobilizando o grupo para reflexão e mudança das práticas de cuidados em saúde.
\end{abstract}

Descritores: Cuidados de enfermagem; educação em enfermagem; dermatite das fraldas; segurança do paciente.

\section{ABSTRACT}

Objective: to evaluate as training a process of educational intervention on incontinence-associated dermatitis with nursing professionals from a public hospital. Method: qualitative, educational intervention study conducted in 2017 with seven internal medicine nursing professionals at a university hospital in the Midwest region of Brazil, using a non-probabilistic convenience sample. The meetings were recorded in their entirety in audio, and the data were treated using the content analysis technique. The study was approved by the research ethics committee. Results: the following thematic categories were identified: epistemological curiosity, research/investigation, evaluation, and joy. Conclusion: the participants reported having understood the subject, and stated that the meetings were conducted efficiently, cheerfully and dynamically. Note that the professionals discussed the problem, which mobilized the group to think about and change health care practices.

Descriptors: Nursing care; education, nursing; diaper rash; patient safety.

\section{RESUMEN}

Objetivo: evaluar de modo formativo un proceso de intervención educativa sobre dermatitis asociada a la incontinencia con profesionales de enfermería de un hospital público. Método: estudio de intervención educativa, con enfoque cualitativo, realizado en 2017, junto a siete profesionales del equipo de enfermería del sector de clínica médica, de un hospital universitario de la región del Centro Oeste de Brasil, siendo la muestra no probabilística por conveniencia. Todos los encuentros fueron grabados en audio y, para el tratamiento de los datos, se utilizó la técnica del análisis de contenido. Estudio aprobado por el Comité de Ética en Investigación. Resultados: por los testimonios, se identificaron las siguientes categorías temáticas: curiosidad epistemológica, encuesta/investigación, evaluación y alegría. Conclusión: los participantes relataron haber comprendido el tema, afirmaron que los encuentros fueron conducidos de forma eficiente, alegre y dinámica. Se resalta que los profesionales realizaron discusiones sobre el problema, movilizando al grupo para la reflexión y el cambio de las prácticas de cuidados en salud.

Descriptores: Atención de enfermería; educación en enfermería; dermatitis del pañal; seguridad del paciente.

\section{INTRODUÇÃO}

A dermatite associada à incontinência (DAI) é uma inflamação cutânea que ocorre em consequência do contato da pele das regiões perineal, perigenital, glúteos, coxas e adjacências com as fezes e/ou urina. As lesões advindas desse contato são caracterizadas por uma hiperemia local ou quando mais graves, por uma ruptura da pele ${ }^{1}$.

\footnotetext{
'Enfermeira. Mestre, Hospital Universitário da Universidade Federal da Grande Dourados. Brasil. E-mail: jaqueline_skm@hotmail.com.

"Enfermeira. Doutora. Professora Titular, Universidade Estadual de Mato Grosso do Sul. Brasil. E-mail: fabiana@uems.br.

I'Enfermeira. Doutora. Professora Titular, Universidade Estadual de Mato Grosso do Sul. Brasil. E-mail: ewatanabe@uems.br.

IVExtraído da dissertação Educação permanente em saúde no cuidado ao cliente com lesão por pressão e dermatite associada à incontinência, apresentada à Universidade Estadual de Mato Grosso do Sul, 2018.
} 
A fisiopatologia do surgimento desta lesão está relacionada à exposição da pele por tempo prolongado a irritantes como fezes e/ou urina. Essa exposição prolongada à umidade altera a função de barreira da pele, levando à um excesso de hidratação, alterando o pH cutâneo e reduzindo sua tolerância à fricção, cisalhamento ou pressão. Além da umidade, as fezes possuem enzimas digestivas que acarretam em maior possibilidade de dano tissular ${ }^{1,2}$.

Com relação aos fatores associados ao surgimento da DAl, pode-se destacar os extremos de idade, o estado geral de saúde do paciente, o estado nutricional, a oxigenação tecidual, a perfusão tissular, a exposição da pele à friç̧ão ou ao cisalhamento e a temperatura corporal. Associado a esses fatores, a existência de incontinência é o fator primordial para o surgimento desta lesão, seja urinária, fecal ou a chamada dupla incontinência. A condição da pele da região perineal, perigenital ou adjacências, a habilidade do paciente de ir ao banheiro sem auxílio, assim como a capacidade cognitiva do mesmo de identificar a necessidade de realizar suas eliminações também são fatores de risco associados ao surgimento da DAl ${ }^{3,4}$.

Esta é uma lesão prevalente em instituições hospitalares, já que rotineiramente nesses locais, os clientes estão em condições patológicas que envolvem o rebaixamento do nível de consciência. Contudo, sua incidência e prevalência ainda não são totalmente esclarecidas. Em meio hospitalar, estima-se uma prevalência de 29,0\% de DAl em clientes incontinentes e uma taxa de $16,5 \%$ na população total de pacientes internados. Outro estudo identificou uma taxa de $21,3 \%$ de DAl em meio hospitalar ${ }^{5,6}$.

No Brasil, um estudo realizado no sudeste do país, identificou uma incidência de 20,4\% de DAl em pacientes internados em unidades de terapia intensiva. A média de tempo entre a internação e o surgimento da lesão foi de nove dias $^{7}$.

A DAl é uma lesão extremamente dolorosa, que reduz a qualidade de vida e o conforto dos pacientes e pode predispor a lesões mais graves, como as lesões por pressão (LPs). Uma DAI não tratada adequadamente e em tempo oportuno, acaba por evoluir para uma LP. Além disso, os profissionais possuem dificuldade em diferenciar a lesão por pressão da dermatite associada à incontinência, já que ambas podem ocorrer em pacientes incontinentes e possuem características semelhantes ${ }^{1,4,8-13}$

Diante de todos esses fatores e da importância da implementação de cuidados adequados para cada uma delas, é indicado que as equipes de saúde sejam capacitadas para realizar o diagnóstico diferencial das mesmas, com o objetivo de aumentar a segurança dos pacientes ${ }^{1,4,8-13}$.

Este estudo teve como objetivo avaliar de modo formativo um processo de intervenção educativa sobre dermatite associada à incontinência com profissionais de enfermagem de um hospital público.

\section{REFERENCIAL TEÓRICO}

No ano de 2004, foi instituída a Política Nacional de Educação Permanente em Saúde (EPS) no Brasil, como uma estratégia para a formação e o desenvolvimento de trabalhadores para o Sistema Único de Saúde. De acordo com esta política, a educação desenvolvida na área da saúde deve agregar o aprendizado juntamente com a reflexão crítica sobre os problemas de saúde vivenciados pelas equipes com o intuito de promover sua resolutividade ${ }^{14}$.

Dentre os pressupostos da EPS, estão a identificação das necessidades educacionais dos trabalhadores da saúde, com a educação sendo desenvolvida no próprio ambiente de trabalho, no quotidiano das instituições. Esses processos educativos devem ter como referência as necessidades de saúde dos sujeitos e das populações atendidas ${ }^{14}$.

Um dos autores utilizados como subsídio para a elaboração da Política Nacional de EPS foi o educador Paulo Freire. Entre os principais pressupostos de sua obra está a importância da educação dialógica, na qual os educandos não são meros ouvintes, mas sim, atores com voz, reflexivos e ativos no processo de ensino-aprendizagem ${ }^{15}$.

Um dos grandes desafios na implementação de processos educativos é a adesão dos participantes às atividades, bem como, o incentivo ao sentimento de pertencimento dos envolvidos no processo. Nesse contexto, a adoção de novas metodologias e o uso de estratégias diversificadas para o ensino podem auxiliar na mudança das práticas de cuidado e promoção de uma assistência livre de danos. É importante também que nos processos educativos os participantes tenham voz e possam sentir-se atores das ações. Outro fator a ser destacado é a aplicabilidade do conteúdo à realidade ${ }^{6,16}$.

Uma metodologia que tem sido adotada na área da saúde é a da problematização. Esta metodologia propõe a execução de cinco etapas para o ensino, sendo elas: a identificação do problema; o levantamento dos pontos-chave relacionados; a teorização, em que ocorre a busca por literatura científica sobre o assunto; a identificação das hipóteses para a solução e, por fim, a aplicação à realidade das soluções encontradas ${ }^{16}$.

Diante do exposto, da interligação direta entre essas lesões (DAl e LP) e da elevada prevalência da DAl em pacientes incontinentes, desenvolveu-se um processo educativo sobre dermatite associada à incontinência, com profissionais de enfermagem atuantes em um setor de clínica médica, pautado na EPS e nos pressupostos de Paulo Freire, valorizando a metodologia dialógica e problematizadora ${ }^{14-16}$. 


\section{METODOLOGIA}

Trata-se de um estudo de intervenção, de abordagem qualitativa. Foi estabelecido através de encontros educativos com profissionais da equipe de enfermagem do Hospital Universitário da Universidade Federal da Grande Dourados (HU-UFGD/Ebserh), em Dourados, MS, Brasil.

Os participantes foram selecionados através de amostra não probabilística por conveniência. Participaram os servidores da enfermagem do período vespertino da clínica médica da instituição. A equipe contava com oito servidores, na época da coleta de dados, que foi realizada em dezembro de 2017. O convite para participar do estudo se estendeu para todos os servidores, porém, devido à demanda de trabalho do setor, sete profissionais concordaram e conseguiram participar do estudo, que totalizou quatro encontros.

Os encontros educativos foram realizados na sala da enfermagem do setor e, para o desenvolvimento do diálogo sobre o tema, os participantes foram dispostos em uma roda, com o intuito de facilitar o debate entre os envolvidos.

Antes do início do estudo, os participantes receberam uma explicação sobre os objetivos da pesquisa, benefícios, riscos e esclarecimento sobre a liberdade em participar ou não da mesma. Somente após consentimento e assinatura individual do Termo de Consentimento Livre e Esclarecido, a atividade foi iniciada.

No primeiro encontro, houve a explanação da pesquisadora sobre a intenção de conduzir encontros educativos com a equipe e, em conjunto, o grupo chegou à escolha do tema DAI. Nesse encontro inicial, foram identificadas as necessidades educativas do grupo sobre a DAI. O delineamento dos momentos educativos está descrito na Figura 1.

\begin{tabular}{|c|c|c|}
\hline $\begin{array}{l}\text { Encontro } \\
\text { educativo }\end{array}$ & Conteúdo abordado nos encontros educativos & $\begin{array}{l}\text { Estratégias de } \\
\text { ensino utilizadas }\end{array}$ \\
\hline 10 & $\begin{array}{l}\text { Definição em grupo sobre o tema e identificação das necessidades educativas através de } \\
\text { tempestade de ideias (Etapas } 1 \text { e } 2 \text {, da Metodologia da Problematização com Arco de } \\
\text { Maguerez) }{ }^{16} \text {. }\end{array}$ & $\begin{array}{l}\text { Tempestade de } \\
\text { ideias }\end{array}$ \\
\hline 2 & $\begin{array}{l}\text { Leitura em grupo de artigos científicos sobre DAl e reflexões sobre os achados (Etapa 3, da } \\
\text { Metodologia da } \\
\text { Problematização com o Arco de Maguerez) }{ }^{16} \text {. }\end{array}$ & Estudo de texto \\
\hline 3으 & $\begin{array}{l}\text { Leitura em grupo de bulas dos produtos disponíveis no mercado e na instituição sobre a DAl, } \\
\text { bem como, realização de aula expositiva dialogada sobre as diferenças das lesões de DAl e LP e } \\
\text { sobre as categorias da DAl (Etapas } 3 \text { e } 4 \text {, da Metodologia da Problematização com o Arco de } \\
\text { Maguerez) }{ }^{16} \text {. }\end{array}$ & $\begin{array}{l}\text { Estudo de texto e } \\
\text { aula expositiva } \\
\text { dialogada com uso } \\
\text { de recursos } \\
\text { multimídia }\end{array}$ \\
\hline 40 & $\begin{array}{l}\text { Uso de jogo educativo para aplicação à realidade do conteúdo discutido durante os encontros } \\
\text { educativos (Etapa } 5 \text {, da Metodologia da Problematização com o Arco de Maguerez) }{ }^{16} \text {. }\end{array}$ & Jogo educativo \\
\hline
\end{tabular}

FIGURA 1: Delineamento dos encontros educativos sobre dermatite associada à incontinência realizados com a equipe de enfermagem. Dourados, MS - Brasil, 2017.

As estratégias de ensino utilizadas foram tempestade de ideias, aula expositiva dialogada, estudo de texto e uso de jogo educativo. Para a criação do jogo, foram selecionados conteúdos de acordo com as evidências científicas sobre DAl e de autores que elaboraram jogos educativos na área da saúde, não ocorrendo a validação do jogo desenvolvido ${ }^{1-}$ 13,17

Neste estudo, adotou-se a Metodologia da Problematização com o Arco de Maguerez para a condução dos encontros educativos ${ }^{16}$. Ao final de cada encontro foi solicitado aos participantes que realizassem uma avaliação do mesmo de modo formativo e individual.

A avaliação formativa tem a intenção de auxiliar na aprendizagem dos alunos, buscando saber dos atores envolvidos, como podem ocorrer melhorias nesse processo. É uma avaliação que ocorre no cotidiano das aulas e ao longo das atividades, com a reflexão sobre elas e de forma dialogada. Esta avaliação pode ser conduzida formalmente ou não ${ }^{18}$.

Os encontros foram gravados em áudio e transcritos na íntegra pela pesquisadora; em seguida foi realizada a análise, com leituras e releituras dos depoimentos, escritos avaliativos dos participantes e dos registros dos diários de campo. Foram abstraídas as unidades de análise, a partir de palavras ou frases significativas e assim, foram identificadas as categorias temáticas, adotando a técnica da análise de conteúdo ${ }^{19}$. De tal análise, emergiram quatro categorias temáticas: curiosidade epistemológica, pesquisa/investigação, alegria e avaliação. 
As falas dos participantes foram identificadas pela letra $P$ (relativa à participante) e seguida do número correspondente à ordem de aparição das falas no primeiro encontro. Desse modo, os atores foram identificados como P1, P2 e assim por diante. Os preceitos éticos foram respeitados, conforme orientações da Resolução no 466/2012 do Conselho Nacional de Saúde. Esta pesquisa recebeu aprovação da Comissão de Ética em Pesquisa da instituição e aprovação do Comitê de Ética em Pesquisa com Seres Humanos da Universidade Estadual de Mato Grosso do Sul, pelo parecer n. 2.197.369 e CAAE: 70593017.8.0000.8030.

\section{RESULTADOS E DISCUSSÃO}

\section{Caracterização dos participantes do estudo}

Os participantes eram profissionais da enfermagem atuantes na clínica médica, setor que assiste pacientes adultos e, em grande parte, dependentes dos cuidados de enfermagem. Dentre os participantes, dois eram enfermeiros e cinco técnicos de enfermagem; cinco do sexo feminino e 5 do sexo masculino. Quanto ao tempo de trabalho na profissão, dois atuavam entre 1-5 anos, dois atuavam entre 5-9 anos e o restante há 10 anos ou mais na enfermagem. Com relação à formação, quatro tinham graduação e três servidores tinham especialização. Nenhum cursou o mestrado ou doutorado.

As categorias temáticas - curiosidade epistemológica, pesquisa/investigação, alegria e avaliação são analizadas a seguir.

\section{Curiosidade epistemológica}

As falas dos envolvidos nos encontros educativos evidenciaram que o estímulo à curiosidade no processo de ensino-aprendizagem é algo necessário e que mobiliza os discentes em busca do saber. No primeiro encontro, os participantes fizeram algumas perguntas que não foram prontamente respondidas, sendo estimulada a leitura de artigos científicos para identificar neles as respostas para as suas indagações ${ }^{20,21}$

Os encontros foram conduzidos de uma maneira simples, porém, dinâmica e instrutiva deixando na expectativa do próximo encontro. (P2)

[...] eu vou pesquisar hoje já. (P1)

Posso usar maisena, talco e/ou AGE para a DAl? (P4)

Ai meu Deus, será que vou ter oportunidade de participar do próximo encontro? (P1)

Ao final do encontro, o grupo parecia não querer encerrar a atividade. No momento em que a pesquisadora informou aos participantes que eles estavam liberados. Os mesmos pareciam não querer dar por encerrado o encontro, mantendo os olhares fixos na pesquisadora. (Diário de campo do pesquisador, encontro 1)

\section{Pesquisa/investigação}

Desde o primeiro encontro o grupo elencou dúvidas sobre a DAI. Visando à elucidação delas, o grupo realizou uma leitura em grupo de artigos e, ao mesmo tempo, ocorreu a discussão e reflexão sobre as práticas ${ }^{3,20,21}$.

Sobre a prevenção: monitorar a integridade da pele; limpar a pele com produtos com $\mathrm{pH}$ acidificado; evitar a fricção ao secar a pele; aplicar emolientes e hidratantes na pele integra; proteger a pele com creme barreira. (P6)

Para a cicatrização, o artigo fala sobre monitorar a evolução da DAl e sua profundidade; limpar a pele com produtos com $\mathrm{pH}$ acidificado ou preferencialmente utilizar toalha impregnada com solução de dimeticona a 3\%. (P6)

O artigo que eu li trata praticamente das mesmas coisas, o que ele diz de diferente é sobre o tratamento. Fala que as medidas de tratamento focalizam a utilização de dietas e exercícios do soalho pélvico para contenção das fezes e urina. Indica também o uso de cremes antifúngicos e corticosteroides tópicos a curto prazo. Para a infecção associada, em casos mais severos, utiliza-se metronidazol $400 \mathrm{mg}$ via oral. (P1)

Como fatores de risco estão a presença de comorbidades; incontinência dupla; elevado nível de dependência para a realização das atividades de autocuidado; pessoas idosas com idade entre 65 anos ou mais. (P1)

Grande interesse do grupo sobre a possibilidade de usar amido de milho e talco na prevenção e tratamento da DAl. Após leitura e discussão de artigos, foi percebido pelo grupo que estes produtos não são indicados. (Diário de campo do observador, encontro 2)

\section{Alegria}

Durante os encontros, foi percebido pela pesquisadora, participantes e observador um clima de descontração. No desenvolvimento das atividades, ocorreram risos, seja por conta de alguma fala colocada ou pela atividade proposta.

Só para fazer bolacha. [respondendo se o amido de milho pode ser usado para o tratamento ou prevenção da $D A l$, no último encontro educativo] (P7) 
Reunião produtiva, trouxe novas terminologias, mais informações e sanou dúvidas de forma dinâmica. (P5)

Foi um encontro agradável com novos conhecimentos em um tema que para mim é novo. (P6)

O grupo como um todo se animou e participou da discussão sobre o tema. (diário de campo do observador, encontro 2)

O processo pedagogico dianalógico e participativo estimula o engajamento discente de maneira prazerosa ${ }^{15,16}$

\section{Avaliação}

Ao final de cada encontro era realizada uma atividade avaliativa do mesmo, de forma escrita, individual e sem identificação, com o intuito de permitir a liberdade dos participantes nesse processo, favorecer a melhoria das atividades desenvolvidas e da pesquisadora como educadora. Eis os discursos obtidos:

Tema bastante relevante pois aborda a vivência cotidiana do posto de trabalho e agrega conhecimentos para melhorar a assistência dispensada ao paciente.

Muito interessante abordar esses temas que estão presentes no nosso dia a dia de trabalho. Precisamos nos atualizar para poder cuidar melhor de nossos clientes.

A importância de trocar a fralda do paciente em curto tempo para evitar a DAI.

$O$ encontro foi produtivo, aprendemos a usar o creme barreira de maneira correta e economizando o material. Melhoramos nossa capacidade de diferenciar LP de DAI.

Achei ótima a dinâmica, os ensinamentos, só assim esclarecemos as dúvidas mais comuns como não poder usar amido de milho ou talco para essas lesões. Infelizmente não estive presente em todos os encontros, pois, estava de folga.

Gostei muito do jogo, ajudou a fixar o conteúdo.

A curiosidade é um fenômeno vital que mobiliza os seres humanos. A curiosidade move os indivíduos e os coloca de forma impaciente diante do mundo. $O$ educador não deve limitar a curiosidade dos seus educandos em nome de uma memorização de conteúdos ${ }^{15}$.

Em conjunto com a curiosidade, inerente ao processo de aprendizado, a alegria também deve estar presente no ato de ensinar e estudar. Parte da responsabilidade de promover essa alegria está no docente, que deve ter alegria ao educar. $\mathrm{O}$ ato de estudar, ensinar e aprender devem ser acessíveis e interessantes. Podem ser exigentes, mas devem ser prazerosos ${ }^{15}$.

Neste estudo, procurou-se atender às curiosidades e dúvidas dos participantes sobre a DAl, assim como, estabelecer um ambiente alegre e descontraído para o diálogo sobre o assunto. As dúvidas estavam relacionadas ao uso de amido de milho e talco para a prevenção ou tratamento da DAl e à diferenciação das lesões semelhantes à DAI, como as LPs. Com relação à possibilidade de utilizar o amido de milho para a DAl, a literatura ressalta que este produto não possui evidência científica para esta finalidade e causa ressecamento da pele, não sendo indicado o seu uso ${ }^{22}$.

Outra dúvida abordada pela equipe estava relacionada ao uso de talco para a prevenção ou tratamento da DAI. O talco é contraindicado para estas lesões por não haver embasamento científico para esse uso, bem como pode propiciar o crescimento de fungos na pele dos pacientes ${ }^{22}$. A equipe também relatou que muitos profissionais fazem uso de AGE para a DAI, tanto para a prevenção, como para o tratamento. Porém, sabe-se que o AGE não é capaz de formar uma camada protetora na pele, sendo facilmente removido ao entrar em contato com a fralda. Além disso, produtos oclusivos não são indicados para estas lesões, assim, o AGE não deve ser utilizado para a DA1 ${ }^{1,23}$.

Cabe ressaltar outra dúvida mencionada pelo grupo - a diferenciação das lesões chamadas confundidoras da DAI, como a LP. Dentre as diferenças dessas lesões pode-se destacar: possibilidade de tecidos desvitalizados na LP, ausência desses tecidos na DAI; a LP é regular em seu formato, a DAl tem formato irregular e difuso; outra diferença é com relação à profundidade, já que uma LP pode ser extremamente profunda e a DAl em geral é superficial. Por fim, outra característica importante está relacionada ao aspecto da hiperemia presente nestas feridas, sendo que na DAl a hiperemia não é uniforme, apresentando vários pontos brancos ou rosados na pele ao redor da lesão e na LP a hiperemia é uniforme ${ }^{24}$.

No desenrolar dos encontros, pode-se observar que o grupo adquiriu novos conhecimentos, como o uso adequado do creme barreira, produto que já existia na instituição, porém, os participantes relataram que não sabiam utilizar o mesmo de modo adequado, aplicando excessivamente o produto e não o distribuindo até desaparecer, após a aplicação na pele. Outra informação relevante é sobre o respeito às orientações do fabricante quanto à periodicidade de troca ou de reaplicação do produto ${ }^{1}$.

A equipe também compreendeu a indicação adequada dos produtos para a DAl. Para a prevenção ou tratamento de uma lesão inicial (DAl categoria 1), pode-se utilizar o creme barreira composto por dimeticona ou pomadas com óxido de zinco. Para lesão já com ruptura da pele (DAl categoria 2), é indicada a película protetora. Caso existam sinais, ao exame físico, de descamação da pele ou prurido, pode-se suspeitar de infecção fúngica associada à lesão, sendo indicado o uso de nistatina associada ao óxido de zinco, por este produto ser capaz de formar uma barreira na pele e 
ter ação antifúngica. Dependendo da gravidade da infecção fúngica, medicamentos orais podem ser associados conforme prescrição médica, sendo normalmente utilizado o metronidazol por via oral ${ }^{1-4,21}$.

Pelas avaliações das atividades foi perceptível o avanço do grupo com relacão ao conhecimento sobre a DAI, relatando modificações e reflexões das práticas até então exercidas. Essas reflexões, são entendidas na metodologia da problematização como o refletir metódico e científico ${ }^{16}$. Foram evidenciadas nas categorias temáticas curiosidade espistemológica e pesquisa/investigação e favoreceram a transformação da prática observada na avaliação.

$\mathrm{O}$ ato de ensinar não deve ser desconectado de alguns aspectos importantes como a pesquisa dos conteúdos e a avaliação do processo educativo, sobretudo quando este é desenvolvido no contexto da prática, no âmbito da EPS. Neste estudo, a pesquisa sobre o assunto foi fundamental para sanar as dúvidas do grupo e fornecer subsídios para uma assistência segura aos pacientes, garantindo o protagonismo, empoderamento e a construção coletiva dos trabalhadores ${ }^{14,25}$.

A metodologia adotada, por meio do Arco de Maguerez, corroborou a reflexão sobre as práticas, por ser uma metodologia problematizadora, que adota o diálogo para promover o ensino e vai além da simples transmissão de conteúdos, permitindo, ainda, a aplicação do conteúdo aprendido ${ }^{16,26}$. Outra experiência semelhante de EPS, por meio da metodologia da problematização, no contexto hospitalar, também corrobora tais achados ${ }^{27}$.

Optou-se por realizar a avaliação formativa para identificar possíveis aspectos relacionados à melhoria do processo conduzido. As falas apresentadas na categoria avaliação foram fruto da avaliação contínua desenvolvida no processo de ensino. Entretanto, entendendo que a avaliação formativa deve ser pautada no diálogo, com a finalidade de construir o saber do aprendiz, em todas as categorias foi possível perceber a construção do conhecimento entre os participantes. Além da avaliação formal, que pode ser necessária para a valoração do conteúdo estudado, é necessário também que o educador tenha um senso de vigilância, percebendo os sinais não verbais durante o ensinar, como o silêncio, um sorriso ou o ato do aprendiz se retirar do ambiente das discussões. Nesse contexto, o espaço pedagógico, onde se realiza o processo de ensino-aprendizagem, deve ser constantemente analisado para identificar se a prática de ensino está sendo efetiva ${ }^{15}$.

Contudo, considerando que este estudo buscou avaliar de modo formativo uma intervenção educativa sobre DAI, acredita-se que as categorias construídas evidenciam a potência do ensino no contexto da prática, principalmente, sobre um tema ainda pouco discutido no cotidiano da enfermagem. O estudo demonstrou as potencialidades de uma avaliação que busca o acolhimento entre os envolvidos, permitindo o diálogo, escuta e confronto honesto, o que favorece o desenvolvimento de mudanças coletivas ${ }^{28}$.

\section{CONCLUSÃO}

O processo de ensino foi realizado dentro do cotidiano de trabalho dos servidores, juntamente com as outras atribuições da equipe, conforme pressupostos da EPS, sendo este um dos desafios vivenciados nesta pesquisa.

Através da análise, identificou-se que a intervenção educativa provocou reflexões, a partir da prática, porém, pautadas no conhecimento científico, e suscitou mudanças no grupo envolvido e tomada de decisão com consciência. Os participantes relataram compreensão do tema e afirmaram que os encontros foram alegres e dinâmicos. Foi perceptível o interesse da equipe durante as atividades, sendo bonito de se ver a tristeza dos participantes por estar de folga em um dos encontros.

Como limitações do estudo, está a amostra, que foi limitada a uma classe profissional. Contudo, a metodologia selecionada e as estratégias adotadas demonstraram ser efetivas para a realização de atividades de ensino em saúde. Espera-se que o processo implementado seja replicado em outros espaços de atenção à saúde para o debate de temas que exijam atualização contínua.

\section{REFERÊNCIAS}

1. Beeckman D, Campbell J, Campbell K, Chimentão D, Coyer F, Domansky R et al. Incontinence-associated dermatitis: moving prevention forward. Wounds int. [Internet], 2015 [cited 2018 May 31]; 1-24. Available from: https://www.woundsinternational.com/resources/details/incontinence-associated-dermatitis-moving-prevention-forward.

2. Collier M, Simon D. Protecting vulnerable skin from moisture-associated skin damage. Br. j. nurs. [Internet], 2016 [cited 2018 May 31]; 25(20):S26-32. DOI: https://doi.org/10.12968/bjon.2016.25.20.S26.

3. Rippon M, Colegrave M, Ousey K. Incontinence-associated dermatitis: reducing adverse events. Br. j. nurs. [Internet], 2016 [cited 2018 Aug 20]; 25(18):1016-21. DOI: https://doi.org/10.12968/bjon.2016.25.18.1016.

4. Voegeli D. Incontinence-associated dermatitis: new insights into an old problem. Br. j. nurs. [Internet], 2016 [cited 2018 Aug 20]; 25(5):256-62. DOI: https://doi.org/10.12968/bjon.2016.25.5.256.

5. Johansen E, Bakken LN, Duvaland E, Faulstich J, Hoelstad HL, Moore Z, et al. Incontinence-associated dermatitis (IAD): prevalence and associated factors in 4 hospitals in Southeast Norway. J. wound ostomy continence nurs. [Internet], 2018 [cited 2019 Jun 20]; 45(6):527-31. DOI: https://doi.org/10.1097/WON.0000000000000480. 
6. Gray M, Giuliano KK. Incontinence-associated dermatitis, characteristics and relationship to pressure injury: a multisite epidemiologic analysis. J. wound ostomy continence nurs. [Internet], 2018 [cited 2019 Jun 20]; 45(1):63-7. Available from: https://www.ncbi.nlm.nih.gov/pubmed/29300291.

7. Chianca TCM, Gonçales PC, Salgado PO, Machado BO, Amorim GL, Alcoforado CLGC. Incontinence-associated dermatitis: a cohort study in critically ill patients. Rev. gaúch. enferm. [Internet], 2016 [cited 2018 Aug 10]; 37(Esp):e68075. DOI: http://dx.doi.org/10.1590/1983-1447.2016.esp.68075.

8. National Pressure Ulcer Advisory Panel, European Pressure Ulcer Advisory Panel, Pan Pacific Pressure Injury Alliance. Prevention and treatment of pressure ulcers: quick reference guide. [Internet]. Perth (AUS): Cambridge Media; 2014. [cited 2018 Aug 13]. Available from: https://proqualis.net/manual/prevenção-de-úlceras-de-pressão-guia-de-consulta-rápido.

9. Beeckman D. A decade of research on incontinence-associated dermatitis (IAD): evidence, knowledge gaps and next steps. J. tissue viability. [Internet], 2017 [cited 2018 Aug 10]; 26(1):47-56. DOI: https://doi.org/10.1016/j.jtv.2016.02.004.

10. Lee YJ, Kim JY, Korean Association of Wound Ostomy Continence Nurses. Effects of pressure ulcer classification system education programme on knowledge and visual differential diagnostic ability of pressure ulcer classification and incontinenceassociated dermatitis for clinical nurses in Korea. Int. wound j. [Internet], 2016 [cited 2018 Aug 10]; S1:26-32. DOI: https://doi.org/10.1111/iwj.12546.

11. Park KH, Choi H. Prospective study on incontinence-associated dermatitis and its severity instrument for verifying its ability to predict the development of pressure ulcers in patients with fecal incontinence. Int. wound j. [Internet], 2016 [cited 2018 Aug 10]; S1:20-5. DOI: https://doi.org/10.1111/iwj.12549.

12. Yates A. Incontinence-associated dermatitis: what nurses need to know. Br. j. nurs. [Internet], 2018 [cited 2019 Jun 10]; 27(19):1094-100. DOI: http://dx.doi.org/10.12968/bjon.2018.27.19.1094.

13. Paulin R, Dowling-Castronovo A. Incontinence-associated dermatitis: it is not a pressure injury. Urol. nurs. [Internet], 2017 [cited 2018 Aug 10]; 37(6):304-9. DOI: https://doi.org/10.7257/1053-816X.2017.37.6.304.

14. Ministério da Saúde (Br). Portaria no 198, de 13 de fevereiro de 2004. Institui a Política Nacional de Educação Permanente em Saúde como estratégia do Sistema Único de Saúde para a formação e o desenvolvimento de trabalhadores para o setor. Brasília (DF): Ministério da Saúde; 2004.

15. Freire P. Pedagogia da autonomia: saberes necessários à prática educativa. 43a ed. São Paulo: Paz e Terra; 2011.

16. Berbel NAN. A metodologia da problematização com o arco de Maguerez: uma reflexão teórico-epistemológica. Londrina (PR): EDUEL; 2012.

17. Silva AKC, Oliveira KMM, Coelho MMF, Moura DJM, Miranda KCL. Development and validation of an educational game for adolescents about breastfeeding. Rev. baiana enferm. [Internet], 2017 [cited 2018 Aug 17]; 31(1):e16476. Available from: https://portalseer.ufba.br/index.php/enfermagem/article/view/16476.

18. Santos L. The relationship between summative and formative assessment: impossibility or challenge? Ensaio: aval. pol. públ. educ. [Internet], 2016 [cited 2018 Aug 17]; 24(92): 637-69. DOI: https://doi.org/10.1590/S0104-40362016000300006.

19. Bardin L. Análise de conteúdo. Lisboa (Pt): Edições 70; 2016.

20. Tavares AP, Ramos AF, Vieira EV, Mendonça S, Fonseca C, Lopes MJ, Mendes FR. Nursing intervention results in the prevention and healing of dermatitis associated with incontinence: a systematic review of the literature. Riase. [Internet], 2016 [cited 2018 Aug 17]; 2(3):708-22. DOI: http://dx.doi.org/10.24902/r.riase.2016.2(3).709.

21. Cunha CV, Ferreira $D$, Nascimento $D$, Felix $F$, Cunha $P$, Penna LHG. Artigo de revisão - dermatite associada à incontinência em idosos: caracterização, prevenção e tratamento. Braz. j. enterestomal ther. [Internet], 2015 [cited 2018 Aug 17]; 13(3). Available from: https://www.revistaestima.com.br/index.php/estima/article/view/218.

22. Black JM, Gray M, Bliss DZ, Kennedy-Evans KL, Logan S, Baharestani MM, et al. MASD part 2: incontinence-associated dermatitis and intertriginous dermatitis. J. wound ostomy continence nurs. [Internet], 2011 [cited 2018 Oct 02]; 38(4):359-70. DOI: http://dx.doi.org/10.1097/WON.0b013e31822272d9.

23. Strehlow BR, Fortes VLF, Amarante MV. Incontinence-associated dermatitis in hospitalized elderly patients: nurses' selfreported knowledge. Rev. pesqui. cuid. fundam. (Online), 2018 [cited 2019 Jun 02]; 10(30):801-9. DOI: http://dx.doi.org/10.9789/2175-5361.2018.v10i3.801-809.

24. Southgate G, Bradbury S. Management of incontinence-associated dermatitis with a skin barrier protectant. Br. j. nurs. [Internet], 2016 [cited 2019 Jun 19]; 25(9):S22-9. DOI: https://doi.org/10.12968/bjon.2016.25.9.S20.

25. Fagundes NC, Rangel AGC, Carneiro TM, Castrol LMC, Gomes BS. Continuing professional development in health for working nurses. Rev. enferm. UERJ. [Internet], 2016 [cited 2019 Oct 10]; 24(1):e11349. DOI: https://doi.org/10.12957/reuerj.2016.11349.

26. Duarte FCP, Goés FGB, Rocha ALA, Ferraz JAN, Moraes JRMM, Silva LF. Preparing for discharge of low-risk newborns to home care. Rev. enferm. UERJ. [Internet], 2019 [cited 2019 Jun 16]; 27(e38523). DOI: https://doi.org/10.12957/reuerj.2019.38523.

27. Flores GE, Oliveira DLL, Zocche DAA. Permanent education in the hospital context: the experience that brings new meaning to nursing care. Trab. educ. saúde. [Internet], 2016 [cited 2019 Oct 10]; 14(2):487-504. DOI: http://dx.doi.org/10.1590/1981-7746sip00118.

28. De Sordi MRL, Lopes CVM, Domingues SM, Cyrino EG. The potential of formative evaluation on the change processes of professional healthcare education. Interface comun. saúde educ. [Internet], 2015 [cited 2019 Oct 10]; 19(SI):731-42. DOI: http://dx.doi.org/10.1590/1807-57622014.1079. 